\title{
Evaluación de un programa de cambio de actitudes con preconductores para prevenir los accidentes de tráfico provocados por el alcohol en Cataluña
}

\section{Evaluation of a program for changing attitudes in pre-drivers to prevent road accidents related to drink-driving in Catalonia}

Laura Arnau Sabatés*; Gemma Filella Guiu*; Mercè Jariot Garcia*; Josep Montané Capdevila*
${ }^{*}$

Dep. de Pedagogía Aplicada, Universitat Autònoma de Barcelona Dep. de Pedagogía y Psicología. Universitat de Lleida

Enviar correspondencia a:

Laura Arnau Sabatés

Universitat Autònoma de Barcelona. Facultat de Ciències de l'Educació.

Edifici G-6 Dep. Pedagogia Aplicada.

08193 Bellaterra (Cerdanyola del Vallès) Catalunya. Espanya.

Tel: +34935811764

Fax: +34 935813052

e-mail: laura.arnau@uab.cat

\section{RESUMEN}

En este estudio se presentan los resultados de una intervención para prevenir el consumo de alcohol en la conducción con preconductores de todo Cataluña. El programa que se aplicó, fundamentado en el cambio de actitudes, pretende reducir considerablemente el peligro que conlleva mezclar el alcohol con la conducción. Para ello se utilizó un diseño clásico cuasi experimental pretest-posttest con grupo control y se aplicaron dos cuestionarios (uno general, con varios factores de riesgo, y otro específico sobre alcohol). El estudio se llevó a cabo con tres grupos: a) experimental 1 , que recibió la aplicación completa del programa, b) experimental 2, que recibió la aplicación de una parte del mismo y c) control, que no recibió los beneficios del programa de reducción del consumo de alcohol. Se constató, a partir del análisis factorial (ACP) del cuestionario específico y de la prueba ANOVA de medidas repetidas, que el grupo experimental 1 presentaba resultados significativamente mejores que los grupos experimental 2 y control, hallándose diferencias significativas entre los hombres y mujeres de la muestra. Los resultados permiten evidenciar que el programa de cambio de actitudes resulta eficaz y que es posible reducir el consumo de alcohol en jóvenes preconductores.

Palabras clave: actitudes, cambio de actitudes, conductores jóvenes, alcohol, reducción de accidentes, seguridad vial.

\section{ABSTRACT}

This study appraises the results of an intervention to prevent drinkdriving in a cohort of pre-drivers in the region of Catalonia (Spain). The program applied, based on attitude change, sets out to reduce significantly the risk of being involved in drink-driving. A classic quasiexperimental pretest-posttest design with control group was used, and two questionnaires were applied: a general one measuring several risk factors, and another one specifically addressing the question of alcohol. The study was carried out with three groups: a) experimental 1, which received the entire program, b) experimental 2 , which received a part of the program, and c) control, which did not receive the benefits of the program. Results from the factor analysis (PCA) and the repeatedmeasures ANOVA suggest that young pre-drivers who received the program obtained better results in road safety and showed less risk of drink-driving than those who did not receive the program or received only part of it. Significant differences were also found between men and women. The results confirm the effectiveness of the attitude-change program and the possibility of reducing alcohol use among young predrivers.

Key words: attitudes, change of attitudes, young drivers, alcohol, traffic accidents, road safety. 


\section{INTRODUCCIÓN}

$\mathrm{E}$ consumo de alcohol es uno de los factores que más influye en el riesgo de accidentes de tráfico. Según la Organización Mundial de la Salud (2009), los conductores más propensos a consumir bebidas alcohólicas antes de conducir son los jóvenes entre 18 y 24 años de edad, especialmente los varones. Los accidentes suelen producirse de noche y generalmente los fines de semana y representan la primera causa de muerte de los jóvenes menores de 30 años. En España, el alcohol fue en 2007 la causa principal del $30.97 \%$ de los accidentes mortales, en porcentajes menores estuvieron presentes el consumo de drogas (10.67\%) y de psicofármacos (6.97\%) (DGT, 2008). El alcohol se considera actualmente como una droga socialmente aceptada y valorada por los adolescentes como un proceso de convertirse en persona (Galduróz y Caetano, 2004). Además, se relaciona con otros comportamientos de alto riesgo como el exceso de velocidad o un uso inadecuado del cinturón (Benson, Mast y Rasmussen, 2000; Fernandes, Hatfield y Job, 2010; Fernandes, Job y Hatfield, 2007).

El endurecimiento de las leyes y las medidas de prevención a nivel estatal y europeo forma parte de la política actual que intenta separar el alcohol de la conducción en preconductores y conductores jóvenes dada la severidad de los accidentes de tráfico en esta franja de edad (OECD, 2006). Para los estados, reducir la conducción con la bebida además de reducir la accidentalidad, repercute en la disminución de la delincuencia y el crimen (Begg, Langley y Stephenson, 2003; Lapham y McMillan, 2006). Una de las estrategias propuestas para reducir los comportamientos de riesgo en la conducción, es intervenir en la formación inicial del conductor mediante programas preventivos sobre beber y conducir (Comisión Europea, 2010; OMS, 2007) y otros relacionados con la deshabituación del alcohol (Cortés y Giménez, 2004). Programas recientemente implementados como el "Safe driver Project" (Aresi, Fornari, Repetto y Scolari, 2009) o "TEND by Night" (Siliquini, Chiadò, Alonso, Druart, Kedzia, Mollica et al., 2010), este último promovido por la Comisión Europea, han demostrado que es posible incidir en las creencias, los hábitos y las actitudes de los jóvenes en relación al consumo de alcohol y otras drogas para que sean conductores más seguros y eficaces.

En Cataluña, por primera vez se introduce un programa preventivo, fundamentado en el cambio de actitudes, de reducción del consumo de alcohol en jóvenes preconductores con ocasión de prepararse para la obtención del permiso de conducir. Su aplicación, en formato de curso-taller, constituye una experiencia innovadora en las autoescuelas catalanas para reducir los accidentes de tráfico y es una práctica que tiende a ser obligatoria para la obtención del certificado oficial de profesor de formación vial, por lo que en Cataluña existe un alto porcentaje de formadores viales, más de 600, preparados para aplicarlo (Arnau y Montané, 2010). De los resultados de este programa, que cuenta con el apoyo del Servei Català de Trànist, dependerá de que se implemente de manera obligatoria en todo el país.
Algunos estudios realizados con jóvenes conductores han constatado la influencia directa y predictiva que tienen las actitudes y sus tres dimensiones (cognición, emociones y conducta) en sus comportamientos de riesgo en la conducción, por encima de la percepción del riesgo y de otros factores de personalidad (OCDE, 2006; Tronsmoen, 2010; Ulleberg y Rundmo, 2003). Sin embargo, para poder desarrollar actitudes positivas y comportamientos favorables hacia la conducción sin la influencia del alcohol, es necesario incidir no sólo en la información o en los procesos de la percepción, sino en los condicionamientos del hábito y en las emociones en relación con este factor de riesgo (Montané, Jariot y Rodríguez Parrón, 2007). Son numerosos los autores que relacionan los procesos de pensamiento con las emociones y los hábitos de comportamiento con el cambio de actitudes (Albarracín, Johnson, Zanna y Kumkale, 2005; Eagly y Chaiken, 1993, 2007; Maio y Haddock, 2010; Montané et al., 2007).

La teoría del cambio de actitudes se relaciona también con la persuasión. Los estudios de Ajzen y Fishbein (2005) constatan como la actitud y la conducta incluyen aspectos cognitivos como el razonamiento, la intención y la planificación, aspectos relacionados con los hábitos y condicionamientos $y$, de alguna forma, también introducen aspectos emotivos que influyen en la previsión del comportamiento. La estructura del afecto descrita en el metanálisis realizado por Armitage y Cooner (2000) y las teorias contemporáneas de la persuasión de Erwin (2001), presentan aspectos relacionados con la planificación de la conducta y los límites del razonamiento de la acción, aspectos íntimamente relacionados con las actitudes y la persuasión.

El programa de sensibilización a preconductores, fundamentado en un modelo de cambio de actitudes propuesto por Montané y Ferrer (1993) y posteriormente, reformulado por Montané et al. (2007), pretende disminuir los accidentes de tráfico en jóvenes preconductores para que conduzcan sin la influencia del alcohol, incidiendo en cada una de las dimensiones de la actitud (cognición, conducta y emociones). Este programa es consistente con los objetivos propuestos en la matriz OEC "Objetivos para la Educación de Conductores" promovida por la Comisión Europea en el marco de las políticas europeas de seguridad vial 2011-2020 (Comisión Europea, 2010)

El programa de reducción del consumo de alcohol para preconductores consta de una secuenciación de cinco fases relacionadas con el cambio actitudinal:

FASE 1. Evaluación inicial: con el fin de conocer el perfil de riesgo- seguridad inicial y preparar la intervención de una manera personalizada

FASE 2. Mejora de la información: permite trabajar la información manera personalizada y grupal. Por ello la información se relaciona con cada uno de los ítems que conforman el cuestionario específico de evaluación inicial.

FASE 3. La revisión de hábitos: contempla hasta qué punto el comportamiento de riesgo en el factor alcohol está instalado y que alternativas existen para transformar los 
comportamientos de riesgo en costumbres y hábitos de seguridad. Se suelen utilizar preguntas como: ¿Qué bebe? ¿Qué cantidad bebe? ¿Cómo lo hace? ¿Con quién lo hace? ¿Qué siente cuando lo hace?

FASE 4. La integración de sentimientos o emociones: incluye la transmisión de emociones, sentimientos y valores relacionados con la comprensión experiencial, presentando víctimas que han sufrido las consecuencias del alcohol en los accidentes de tráfico. Estas emociones ayudan a fortalecer las ideas y a aceptar los nuevos hábitos de conducción.

FASE 5. La evaluación final: permite detectar a corto y medio plazo las ganancias obtenidas al comparar las diferencias entre la evaluación inicial y la final después del desarrollo del proceso formativo.

La secuenciación establecida por las cinco fases del modelo de cambio de actitudes condiciona tanto el diseño, como la aplicación y evaluación del programa. La eficacia de dicho modelo, que tiene como objetivo último la "tolerancia cero" a la accidentalidad, ha sido constatada en diferentes estudios realizados en Cataluña con preconductores y conductores temerarios (Arnau y Montané, 2010; Jariot y Montané, 2009; Montané y Ferrer, 1993; Montané et al., 2007).

El objetivo de este estudio es evaluar la eficacia de un programa de sensibilización, fundamentado en el cambio de actitudes, aplicado a una muestra de preconductores para prevenir el consumo de alcohol en la conducción. Se espera que los preconductores que han recibido la aplicación completa del programa obtengan mejores resultados que aquéllos que sólo lo han recibo parcialmente o bien han seguido un proceso de formación tradicional.

\section{MÉTODOS}

\section{Participantes}

El total de preconductores que participaron en el estudio experimental fue de 348 . Fueron asignados de forma estratificada y proporcional en función de las variables demarcación, tipología de autoescuela, edad y sexo. Sin embrago, sólo 61 recibieron la aplicación del programa específico de alcohol y conducción, ya que su aplicación depende de los resultados obtenidos en el perfil general de seguridad-riesgo del preconductor que muestra el QAR-Precon y de la necesidad de reducir esta problemática específica. Este grupo denominado experimental 1, que ha seguido el programa con la secuenciación de las cinco fases que establece el modelo de cambio de actitudes, está conformado por 30 mujeres (49 $\%)$ y 31 hombres (51\%).

Para la selección del grupo control se pidió la colaboración a 12 formadores viales pertenecientes, fundamentalmente, a autoescuelas de la provincia de Barcelona para que aplicaran en las clases teóricas y dentro de un intervalo similar al de la aplicación del programa, el pretest y posttest del cuestionario específico sobre alcohol. 32 hombres (56.1\%) y 25 mujeres (43.9\%) forman parte de los 57 sujetos que, aún presentando un nivel de riesgo moderado-alto en el QARPrecon, no recibieron la aplicación del programa específico y se limitaron a seguir el proceso de formación tradicional.

Para delimitar el grupo experimental 2, se utilizó una muestra de oportunidad que permitió incorporar al estudio 41 sujetos preconductores pertenecientes a las diferentes demarcaciones catalanas. Este grupo, formado por 26 mujeres (53.1\%) y 23 hombres (46.9\%) sólo recibió la aplicación de una de las cinco fases del programa de sensibilización, concretamente la mejora de la información, en la que se incluyeron elementos de persuasión y convicción relacionados con las emociones pero sin la presencia directa de la víctima.

Los tres grupos de sujetos presentan edades mayoritariamente comprendidas entre los 18 y 24 años de edad. En los tres grupos el único criterio de selección fue el perfil inicial de riesgo en relación con el alcohol. No obstante, en el grupo experimental 2, por circunstancias de intervención, los formadores participantes aplicaron una dinámica grupal y no el QAR-Precon para dictaminar el perfil inicial de riesgo de los preconductores y su posterior inclusión en el estudio.

\section{Instrumentos}

Los cuestionarios aplicados fueron dos. El primero fue el Cuestionario de Evaluación del Riesgo para Preconductores (QAR- Precon) para poder detectar los indicadores de riesgo en el inicio de la conducción, permitiendo la selección del programa específico. Dicho cuestionario, elaborado por Montané, Jariot y Rodríguez Parrón (2006) presenta un índice elevado de fiabilidad (.095) y de validez externa (.45). Consta de 90 ítems que analizan conocimientos, comportamientos y emociones. Está dividido en tres bloques: salud y cuidado personal con 6 ítems, los grandes factores de riesgo con 75 ítems y el test de madurez de la conducción eficaz con 9 items.

El otro instrumento utilizado fue el cuestionario específico de alcohol, diseñado ad hoc, para evaluar de manera más concreta el perfil de riesgo de los preconductores puesto que sólo recoge información relacionada con el consumo de alcohol y la conducción. Este cuestionario consta de 9 ítems que analizan falsas creencias e intenciones de comportamiento. Se responde en una escala Likert de 4 puntos.

\section{Procedimiento}

Se aplicó un diseño pretest-posttest con grupo control. Para el análisis de los datos se utilizó el programa estadístico SPSS (Statistical Package for Social Sciences). Así, en primer lugar, se realizó un análisis de la estructura interna del cuestionario específico de alcohol mediante la técnica de análisis 
de componentes principales (ACP) con el método de rotación varimax, y se calcularon los coeficientes de fiabilidad mediante el procedimiento reliability. Además, se realizó un análisis de varianza, concretamente el procedimiento modelo lineal general con medidas repetidas, para estudiar el efecto del programa de cambio de actitudes en cada uno de los tres grupos, considerando la variable sexo.

\section{RESULTADOS}

\section{Validez del cuestionario específico}

Los resultados de la medida de adecuación muestral de Kaiser-Meyer-Olkin (.82) y la prueba de esfericidad de Barlett $(p<.001)$ permiten concluir que la extracción factorial resulta pertinente. La rotación con el método varimax, que ofrece 2 autovalores mayores que la unidad, siendo dominante el primero, converge en 3 iteraciones y tiene una interpretación significativa que explica el $57.1 \%$ de la varianza del cuestionario específico sobre alcohol (Tabla 1). La estructura bidimensional extraída se corresponde con dos dimensiones del alcohol postuladas teóricamente y necesarias para mejorar las actitudes de los jóvenes en relación al consumo de alcohol y la conducción, lo que supone una constatación empírica de las mismas.

La matriz de cargas factoriales (Tabla 1) muestra que todas las variables del cuestionario saturan en uno de los dos factores, si bien algunas saturan similarmente sobre dos factores: tal es el caso de los ítems 1, 6 y 8 que están relacionados con lo que miden ambos factores.
El primer factor explica un $44,74 \%$ de la varianza y el segundo un $12,41 \%$. A continuación se presentan las características más relevantes de los 2 factores extraídos:

El primer factor agrupa cinco ítems $(3,4,6,7,9)$ relacionados con la información errónea o la falta de información que los preconductores tienen en relación al alcohol, tales como: "Conozco los efectos de las drogas en la conducción y que creo que no me afectarán cuando conduzca" y "Creo que beber un poco me da energías y me permite conducir mejor". Es por ello que se denomina "Falsas creencias relacionadas con el alcohol".

El segundo factor recoge 4 ítems $(1,2,5,8)$ que se refieren a los hábitos e intenciones de conducir bajo los efectos del alcohol, por lo que se propone denominarlo "hábitos e intenciones de comportamiento relacionadas con el alcohol y la conducción"; entre los ítems con más saturación destacan: "Puedo o podré conducir con seguridad con dos cervezas, dos vasos de vino y/o dos copas de cava" y "Estoy dispuesto a no beber para acompañar a mis amigos con el coche con seguridad".

Ambos factores, considerados como variables dependientes o respuesta, muestran una correlación positiva modera$d a$, estadísticamente significativa $(r=.634, p<.001)$, por lo que los preconductores que puntúan alto en el factor falsas creencias también lo hacen en el de hábitos e intenciones de comportamiento, sin existir ambivalencia en su predisposición actitudinal (Fabrigar, MacDonald y Wegener, 2005).

Asimismo, se comprobó la validez concurrente (convergente) de la escala específica, utilizando como referente el bloque de ítems de alcohol del cuestionario global QAR Precon. La correlación de Pearson entre los dos cuestionarios es de .271 ( $p<.05)$ lo que muestra una correlación estadís-

Tabla 1. Análisis factorial exploratorio de componentes principales del cuestionario específico de alcohol

Análisis factorial exploratorio de componentes principales con rotación varimax (se han omitido las cargas inferiores al valor 0.3)

\begin{tabular}{|c|c|c|}
\hline \multirow[t]{2}{*}{ Ítems } & \multicolumn{2}{|c|}{ Factores } \\
\hline & 1 & 2 \\
\hline 3. Creo que con el alcohol que consumo, cuando conduzca, no afectará a mi seguridad. & .689 & \\
\hline 4. Creo que beber un poco me da energías y me permite conducir mejor. & .493 & \\
\hline 6. Sé lo que tengo que hacer para que desaparezcan los síntomas del alcohol antes de conducir. & .610 & .549 \\
\hline 7. Conozco los efectos de las drogas en la conducción & .830 & \\
\hline 9. Creo que en un futuro si tengo que conducir no tomaré ningún tipo de droga, son peligrosas. & .749 & \\
\hline 1. Aguanto bien el consumo de bebidas alcohólicas y puedo/podré conducir con seguridad. & .534 & .543 \\
\hline 2. Puedo o podré conducir con seguridad con dos cervezas, dos vasos de vino y/o dos copas de cava. & & .867 \\
\hline 5. Estoy dispuesto a no beber para acompañar a mis amigos con el coche con seguridad. & & .540 \\
\hline 8. Cuando tome algún tipo de droga conseguiré que no me afecte en la conducción & .529 & .616 \\
\hline
\end{tabular}

Nombres de los factores: 1 . Falsas creencias sobre el alcohol y otras drogas; 2 . Hábitos e Intenciones de comportamiento 
ticamente significativa, aunque moderada, entre ambos instrumentos. Con lo que se puede afirmar que el cuestionario específico de alcohol posee validez concurrente.

\section{Fiabilidad del cuestionario específico}

La fiabilidad total del cuestionario específico sobre alcohol presenta un nivel bueno y aceptable de consistencia interna $(\alpha=0,81)$. El factor intenciones de comportamiento presenta mejor fiabilidad $(\alpha=0,77)$ que el factor falsas creencias $(\alpha=0,69)$ si bien en ambos es elevada aunque inferior al alfa global. Se puede afirmar que el cuestionario, de manera general, presenta un nivel alto y aceptable de fiabilidad y de consistencia interna.

\section{Efecto del programa de intervención fundamentado en el cambio de actitudes}

Para conocer el efecto del programa de sensibilización alcohol y conducción, el primer paso ha sido comprobar si las matrices de covarianzas son iguales para los tres grupos en el factor general del alcohol. Los resultados obtenidos en el estadístico M. de Box no permiten aceptar la hipótesis de igualdad entre matrices de varianzas - covarianzas, dado que la significación es $p<0.001$.
Los preconductores de los grupos experimental 1 y control presentan un nivel moderado-alto de riesgo en el consumo de alcohol recogido en el cuestionario específico, sin existir entre ellos diferencias iniciales estadísticamente significativas. Se trata de grupos homogéneos en lo relativo a las variables del cuestionario. No obstante el grupo experimental 2 presenta diferencias iniciales significativas respecto con los grupos experimental 1 y control con una diferencia de medias de -.22 ( $p=.038)$ y de $-.33(p<.001)$ respectivamente, siendo el experimental 2 el que presenta un nivel de riesgo menor. Esta variación inicial se debe, probablemente, al proceso de selección del mismo, y a la inclusión adicional, según los formadores viales, de algunos sujetos interesados en recibir información relacionada con el alcohol pese a no presentar un riesgo alto en este factor.

Para comprobar la evolución de la variable "alcohol" en los tres grupos (experimental 1, experimental 2 y control) se ha realizado un ANOVA mixto $(2 \times 2 \times 3)$ siendo el grupo y el sexo variables entre sujeto y el pretest-posttest medidas repetidas. A la vista de los resultados presentados en la tabla 2 , se puede comprobar que la evolución de la variable alcohol es distinta según el grupo al que pertenecen los sujetos que han participado en esta investigación:

En el modelo general, se destaca que la interacción "alcohol - grupo", es significativa ( $p<0.001)$ no así, las interacciones "alcohol - sexo" y "alcohol-sexo- grupo". El tamaño

Tabla 2. Pruebas de los efectos intra-sujetos e inter-sujetos en el factor general del alcohol

\begin{tabular}{|l|l|c|c|c|}
\hline \multicolumn{5}{|c|}{ MODELO 1. Factor alcohol general } \\
\hline Pruebas Efectos & Fuente & $\mathrm{F}$ & $p$-valor & Eta $^{2}$ parcial \\
\hline \multirow{3}{*}{ intra-sujetos } & Factor & 163.761 & $<.001$ & .500 \\
\cline { 2 - 5 } & Factor ${ }^{*}$ Grupo & 30.044 & $<.001$ & .267 \\
\cline { 2 - 5 } & Factor Sexo $^{*}$ & .222 & .638 & .001 \\
\cline { 2 - 5 } & Factor Sexo* Grupo & .207 & .813 & .003 \\
\hline inter-sujetos & Grupo & 12.779 & $<.001$ & .137 \\
\cline { 2 - 5 } & Sexo & 9.168 & $<.005$ & .054 \\
\hline
\end{tabular}

del efecto (Eta al cuadrado parcial) resulta bajo (<.20) en casi todos los casos siguiendo la interpretación de Cohen (1988).

Analizando las diferencias de medias intragurpos se detecta claramente que el nivel de riesgo en el grupo experimental 1 en relación con el alcohol disminuye de manera significativa una vez finalizado el programa, con una diferencia de medias de .71 ( $p$ <.001). También se observa una disminución de las puntuaciones en los grupos control y experimental 2 si bien éstas no son estadísticamente significativas. Gráficamente, también se pueden apreciar tales diferencias (Figura 1):
En los contrastes a posteriori (Bonferroni) se observa que entre el grupo experimental 1 y el grupo control, en el posttest, existen diferencias significativas ( $<.001)$, con una diferencia de medias de -.53 , al igual que entre los grupos experimental 1 y experimental 2 dónde la diferencia significativa se sitúa en -.37 ( $p<.001)$, no así entre los grupos control y experimental 2 dónde las variaciones en el posttest no son estadísticamente significativas ( $p=.057)$, puesto que no se producen mejoras destacables y la diferencia de medias tan sólo es de .16. En este sentido y atendiendo al nivel de significación que muestra $p$ valor en este factor $(F=12.779$; 


\section{Medias marginales estimadas de MEASURE_1}

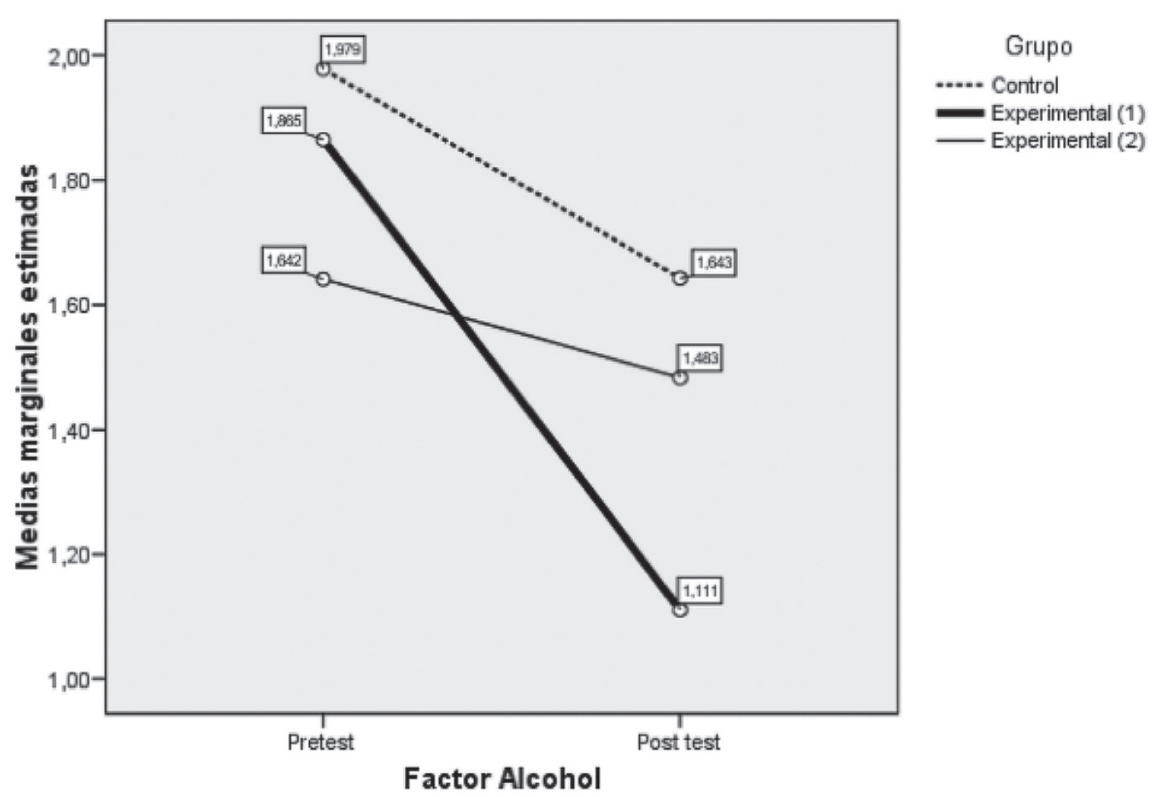

Figura 1. Efecto del programa de cambio de actitudes en relación con el alcohol, por grupos

$p<.001)$, se detectan diferencias evidentes entre el grupo experimental 1 y los grupos experimental 2 y control.

Existen variaciones significativas en los contrastes preposttest en términos de género pese a presentar, tanto hombres como mujeres, una evolución parecida en función del tratamiento recibido. Tal y como puede observarse gráfica- mente (Figuras 2 y 3), los hombres de la muestra presentan, en los tres grupos, un mayor nivel de riesgo en el consumo de alcohol y conducción comparativamente con las mujeres, siendo esta diferencia significativa $(F=9.168 ; p<.005)$.

Pese a las diferencias iniciales intergrupos, se observa claramente que el nivel de riesgo en el grupo experimental 1

\section{Medias marginales estimadas de MEASURE_1}

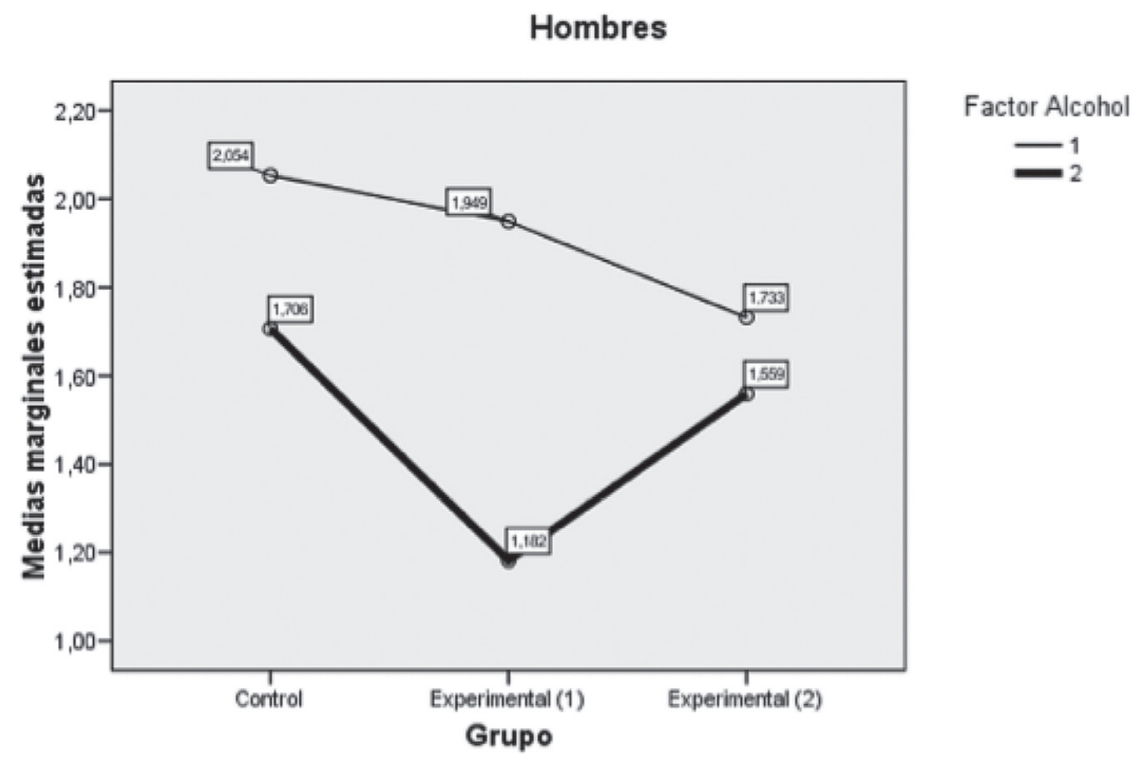

Factor Alcohol 1 = puntuaciones pretest $\cdot$ Factor Alcohol 2 = puntuaciones posttest

Figura 2. Efecto del programa de cambio de actitudes en relación con el alcohol, por sexos (hombres) 


\section{Medias marginales estimadas de MEASURE_1}

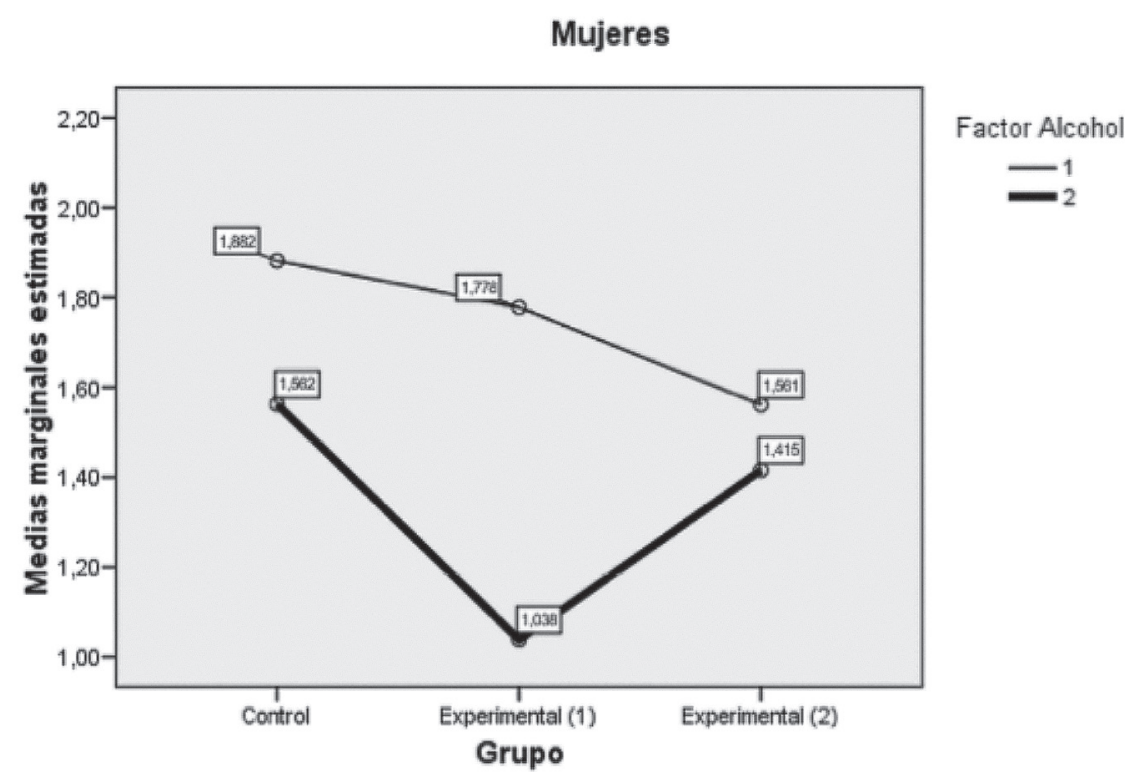

Factor Alcohol 1 = puntuaciones pretest - Factor Alcohol 2 = puntuaciones posttest

Figura 3. Efecto del programa de cambio de actitudes en relación con el alcohol, por sexos (mujeres)

disminuye después de la realización del programa de cambio de actitudes, tanto en hombres como en mujeres, siendo las mujeres las que presentan un nivel de riesgo menor en los tres grupos. Se observa también que el nivel de riesgo casi no disminuye en situación posttest en los grupos control y experimental 2 y que esta estabilidad en las medidas pre-posttest afecta a hombres y mujeres.

\section{DISCUSIÓN Y CONCLUSIONES}

Los datos relacionados con la eficacia del programa de intervención demuestran que existen diferencias significativas en el grupo experimental 1 entre la evaluación realizada en el pretest y la desarrollada en el posttest en el factor alcohol. No obstante, en los grupos control y experimental 2 se presentan diferencias no significativas entre la evaluación realizada en el pretest y la aplicada en el posttest en dicho factor, por lo que las diferencias obtenidas pudieron ser debidas simplemente al azar del muestreo. Estos resultados coinciden con investigaciones anteriores (p.e., Jariot y Montané, 2009).

Los resultados obtenidos señalan diferencias significativas entre hombres y mujeres, siendo los hombres los que presentan, en los tres grupos, un nivel de riesgo mayor en situación de pre-posttest. Estos datos son consistentes con las investigaciones realizadas por Sirkku y Keskinen (2008) y Fernandes et al. (2010), que apuntan un mayor riesgo en el consumo de alcohol y conducción en el caso de los varones jóvenes.
A nivel general, podemos afirmar que un programa de estas características, aplicando la secuencia de las cinco fases que establece el modelo de cambio de actitudes, mejora, de manera efectiva, el nivel de seguridad de los preconductores que reciben su aplicación, tanto hombres como mujeres, reduciendo el riesgo de accidentes de tráfico provocados por el alcohol. En este sentido, parece posible mejorar las actitudes de los preconductores frente al consumo de alcohol y conducción, especialmente las de aquéllos que reciben la aplicación completa y secuenciada del programa de sensibilización.

De los resultados empíricos se desprende que para conseguir mejorar las actitudes de los jóvenes es necesario aplicar las cinco fases del modelo de cambio de actitudes (evaluación inicial, mejora de la información, revisión de hábitos, integración de emociones y evaluación final). En este sentido, la aplicación correcta de las 5 fases produce mejores resultados que si se mezcla la fase de información con elementos de persuasión y sin la influencia de las víctimas de tráfico, procedimiento seguido por el grupo experimental 2 .

De esto se deduce que el proceso formativo para el cambio de actitudes relacionado con el consumo de alcohol y la conducción debe integrar: la información necesaria, la revisión de hábitos de riesgo para que los jóvenes adquieran conciencia de su importancia y se propongan substituirlos por hábitos más seguros y finalmente, la participación de testimonios víctimas de accidentes de tráfico, para que los preconductores integren emociones y sentimientos relacionados con las consecuencias de los accidentes causados por el alcohol. Tanto la información como los hábitos de comportamiento están motivados por lo que sienten los preconduc- 
tores y por las consecuencias negativas que puede acarrear su conducta temeraria. El componente afectivo, de acuerdo con Sjöberg (1998, 2006), Iversen y Rundmo (2004) y Montané et al. (2007), ayuda a dar sentido a la información y a los hábitos de comportamiento.

En consonancia con Montané et al. (2007) la información por si sola (aspecto que se trabaja en el proceso de formación tradicional) no ayuda suficientemente a integrar nuevos hábitos ni a mejorar las emociones. Desde esta perspectiva, la formación para el cambio de actitudes se limita a impartir conocimientos y modificar creencias a base de transmitir información, a través de la elaboración de mensajes convincentes, esperando que ésta influya en la conducta. No obstante, para desarrollar actitudes positivas en la conducción y comportamientos favorables a no beber si se tiene que conducir, será necesario incidir no sólo en la información o en los procesos lógicos y perceptivos, sino en los condicionamientos del hábito y en las emociones. En este sentido, la aplicación de un programa de estas caracteristicas permitiría reforzar y enriquecer el propio proceso de formación tradicional que siguen los preconductores en las autoescuelas catalanas.

Los resultados confirman empíricamente la teoría expuesta en la fundamentación científica, constatando la necesidad de integrar en un programa de intervención los tres elementos (emociones, cognición y conducta) para lograr un cambio de actitudes eficaz (Eagly y Chaiken, 2007; Albarracín et al., 2005; Maio y Haddock, 2010).

En sintesis, la aplicación de la secuencia de las cinco fases del modelo de cambio de actitudes ayuda a mejorar el nivel de riesgo en relación con el alcohol, no así la aplicación asistemática y/o parcial de dicho modelo.

Una de las principales limitaciones del presente estudio es el tamaño de la muestra. Las tendencias apuntadas podrian confirmarse con una eventual ampliación de la misma. Sin embargo, la muestra de la que disponemos permite conocer, de manera preliminar, los efectos del programa de cambio de actitudes en el alcohol aplicado por primera vez en Cataluña y realizar una evaluación preliminar. Los resultados del grupo experimental 2 deben interpretarse con cautela ya que probablemente podrian estar sesgados, pues representan a una submuestra expuesta a menor riesgo, manteniendo diferencias iniciales y de intervención importantes con los grupos experimental 1 y control. Por lo que habrá que ser prudentes y entender que la explicación del efecto diferencial de los resultados del programa en cada uno de los grupos será sólo generalizable en el caso de que la población integre las variables básicas de la muestra utilizada. Finalmente, el proceso de aplicación y de respuesta de ambos cuestionarios puede estar influenciado por la deseabilidad social. Sin embargo, para minimizar tal efecto se pidió a los participantes que contestaran con sinceridad y se mantuvo el anonimato de los dos cuestionarios tal y como sugieren Zhao, Mann, Chipman, Adlaf, Stoduto y Smart (2006), utilizando códigos de asignación numérica. Algunos estudios relacionados con la medición de actitudes de riesgo en la conducción confirman la precisión de las encuestas en forma de cuestionario en este campo (Lajunen \& Summala, 2003; Iversen, 2004; Tronsmoen, 2010)

Con la intención de corroborar los resultados obtenidos, resultaría positivo aplicar el programa de sensibilización alcohol y conducción a diferentes grupos de edad y a diferentes colectivos de conductores. Por otra parte, un estudio longitudinal permitiría constatar la eficacia del programa en relación con las variables sexo y edad y confirmar las tendencias apuntadas entre hombres y mujeres, ajustando, si fuera necesario, el programa para responder de diferente manera sobre cada uno de estos colectivos.

\section{AGRADECIMIENTOS}

Los autores quieren agradecer al Servei Català de Trànsit y a la Càtedra de Formació i Educació Viàries por su apoyo en este estudio.

\section{REFERENCIAS}

Ajzen, I. y Fishbein, M. (2005). The Influence of Attitudes on Behavior En D. Albarracin, B.T. Johnson y M.P. Zanna (Eds.), The Handbook of Attitudes (pp.173-222). Mahwah, NJ: Lawrence Erlbaum Associates, Inc.

Albarracin, D., Johnson, B.T., Zanna, M.P. y Kumkale, G.T. (2005) Attitudes: Introduction and Scope. En D. Albarracin, B.T. Johnson, y M.P. Zanna (Eds.), The Handbook of Attitudes (pp.3-20). Mahwah, NJ: Lawrence Erlbaum Associates, Inc.

Aresi,G., Fornari, L., Repetto, C. y Scolari, M. (2009). Evaluación de una intervención con conductores designados para prevenir los accidentes de tráfico provocados por el alcohol en las discotecas de Milán, Italia. Adicciones, 21, 279-288.

Armitage, C. J. y Conner, M. (2000). Attitudinal ambivalence: A test of three key hypotheses. Personality and Social Psychology Bulletin, 26, 1421-1432.

Arnau, L. y Montané, J. (2010). Indicadores para mejorar el desarrollo profesional de los aspirantes a profesores de formación vial en Cataluña. Revista Española de Orientación y Psicopedagogía, 21(2), 295-307.

Begg, D.J., Langley, J.D. y Stephenson, S. (2003). Identifying factors that predict persistent driving after drinking, unsafe driving after drinking, and driving after using cannabis among young adults. Accident Analysis and Prevention,35, 669-675.

Benson, B.L., Mast, B.D. y Rasmussen, D.W. (2000). Can Police Deter Drunk Driving? Applied Economics, 32(3), 357-66.

Cohen, J. (1988). Statistical power analysis for the behavioural sci-

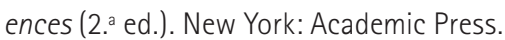

Comisión Europea (2010). Hacia un espacio europeo de seguridad vial: orientaciones políticas sobre seguridad vial 2011-2020. Bruselas: Comisión Europea. 
Cortés, M.T. y Giménez, J.A. (2004). Posibles actuaciones psicológicas para reducir la conducción bajo los efectos del alcohol y viabilidad de su aplicación en España. Adicciones, 16, 97-108.

DGT (2008) Las principales cifras de la siniestralidad vial. Madrid: DGT.

Eagly, A.H. y Chaiken, S. (1993). The Psychology of Attitudes. Fort Worth, TX: Harcourt Brace.

Eagly, A. y Chaiken, S. (2007). The advantages of an inclusive definition of attitude. Social Cognition, 25, 582-602.

Erwin, P. (2001). Attitudes and persuasion. New York: Psychology Press.

Fabrigar, L.R., MacDonald, T.K. y Wegener, D.T. (2005). The Structure of Attitudes. En D. Albarracin, B.T. Johnson y M.P. Zanna (Eds.), The Handbook of Attitudes (pp.79-124). Mahwah, NJ: Lawrence Erlbaum Associates, Inc.

Fernandes, R., Job, R.F.S. y Hatfield, J. (2007). Different factors predict different risky driving behaviours: A challenge to the assumed generalizability of prediction and countermeasure. Journal of Safety Research, 38(1), 59-70.

Fernandes, R., Hatfield, J. y Job, R.F.S. (2010). A systematic investigation of the differential predictors for speeding, drink-driving, driving while fatigued, and not wearing a seat belt among young drivers. Transportation and Research Part F, 13, 179-196.

Galduróz, J.C. y Caetano, R. (2004). Epidemiología del consumo de alcohol en Brasil. Revista Brasileira de Psiquiatria, 26 (suppl.1), 3-6.

Iversen, H. (2004). Risky-taking attitudes and risky driving behaviour. Transportation and Research Part F, 7, 135-150.

Iversen, H. y Rundmo, T. (2004). Attitudes towards traffic safety, driving behaviour and accident involvement among the Norwegian public. Ergonomics, 47, 555-572.

Jariot, M. y Montané, J. (2009). Actitudes y velocidad en jóvenes. Aplicación de un programa de educación vial. Relieve, 15(1).

Lajunen, T. y Summala. H. (2003). Can we trust self-reports of driving? Effects of impression management on driver behaviour questionnaire responses. Transportation Research Part F: Traffic Psychology and Behaviour, 6, 97-107.

Lapham, S. y McMillan, G.P. (2006). Effectiveness of bans and laws in reducing traffic deaths: Legalized Sunday packaged alcohol sales and alcohol-related traffic crashes and crash fatalities in New Mexico, American Journal of Public Health, 96(11), 1944-1948.

Maio, G. y Haddock, G. (2010). The psychology of attitudes and attitude change. London, UK: SAGE Publications Ltd.

Montané, J. y Ferrer, F. (1993). Cuaderno de formación de formadores del conductor: Programa de educación del conductor. Barcelona: PPU.

Montané, J., Jariot, M. y Rodriguez Parrón, M. (2006). Qüestionari d'avaluació del risc d'accidents de trànsit dels conductors. QARC. Barcelona: Servei Català de Trànist.

Montané, J., Jariot, M. y Rodríguez Parrón, M. (2007). Actitudes, Cambio de Actitudes y Conducción segura: Un enfoque crítico aplicado a la reducción de accidentes. Barcelona: Laertes.

OECD. (2006). Young Drivers. The Road to Safety. Paris: OECD Publishing.

OMS (2009). Informe sobre la situación mundial de la seguridad vial: es hora de pasar a la acción. Ginebra: ediciones OMS.

Siliquini, R., Chiadò, S., Alonso, F., Druart, A., Kedzia, M., Mollica, A.,... Manzoli, L. (2010). A European study on alcohol and drug use among young drivers: the TEND by Night study design and methodology. BMC Public Health, 10, 205.

Sirkku, L. y Keskinen, E. (2008). Fatal Drink-Driving Accidents of Young Adult and Middle-Aged Males-A Risky Driving Style or Risky Lifestyle? Traffic Injury Prevention, 9, 195-200.

Sjöberg, L. (1998). Worry and risk perception. Risk Analysis, 18, 85-93.

Sjöberg, L. (2006). Will the Real Meaning of Affect Please Stand Up? Journal of Risk Research, 9(2), 101-108.

Tronsmoen, T. (2010). Associations between driver training, determinants of risky driving behaviour and crash involvement. Safety Science, 48, 35-45

Ulleberg, P. y Rundmo, T. (2003).Personality, attitudes and risk perception of risky driving behaviour among young drivers. Safety Science, 41, 427-443.

Zhao, J. Mann, R.E., Chipman, M., Adlaf, E., Stoduto, G. y Smart, R.G. (2006). The impact of driver education on self -reported collisions among young drivers with a graduate licence. Accident Analyses and Prevention, 38, 35-42. 
